\title{
Two Hypothesis on a Combinatorial Problem for Possible States on the Arrival Line for $\mathbf{n}$ Competitor Runners
}

\author{
Nicolae Popoviciu \\ Department of Informatics, Faculty of Informatics, Hyperion University, Bucharest, Romania
}

Email address:

popoviciunicolae15@yahoo.ro

\section{To cite this article:}

Nicolae Popoviciu. Two Hypothesis on a Combinatorial Problem for Possible States on the Arrival Line for n Competitor Runners. Applied and Computational Mathematics. Vol. 10, No. 3, 2021, pp. 46-55. doi: 10.11648/j.acm.20211003.11

Received: April 9, 2021; Accepted: May 25, 2021; Published: June 22, 2021

\begin{abstract}
In a very small t-time interval, several runners could occupy the same place on the arrival line (hypothesis 1). Each runner has his own name and a competition number (on the shirt). The number of runners is a natural number $\mathrm{n}$. For each given $\mathrm{n}$, the hypothesis creates a combinatorial problem having a lot of posible states. All notations are choose so that to indicate easily by name their meaning. The states are separated into two classes: non-nominal states and nominal states. The states are related with the place I, II, III etc on arrival line. It is necessary to generate the total number of non-nominal states (on arrival line) and the total number of nominal states. In order to generate the states the work uses some formulas and some specialised algorithms. For example, the consrtuction of all non-nominal states recommends that the string for the position I to use a decreasing string. The same rule is validly for position II, but for sub-strings etc. A lot of numerical examples ilustrate the states generation. An independent method verifies the correctitude of states generation. In order to continue the study of combinatorial problem, the work introduces two new notions in section 5. The notions of partial frequency and final frequency are defined for a nominal known runner in final classification, together with computational formulas. The section 6 constructs the random variables attached to final classification and the probability of each place on arrival line. Each runner receives a score (a number of points) related with his final classification. May be the runner is interested to know the probability to ocuppy the first place (place I) and to estimate the number of possible points. All the results could be written in a centralisation table (section 7). Section 8 contains several numerical examples with statistical computations. At the end of the work we replace hypothesis 1 by hypothesis 2 : only one runner could ocuppay each place. All the above notions have a new specific form. The numerical examples ilustrates the theory.
\end{abstract}

Keywords: Arrival Line, Non-nominal State, Nominal State, Partial Feequency, Final Frequency, Algorithm of Arrival Line, Combinatorial Problem, Statistical Problem

\section{Problem Formulation and Special Notations}

A number of $n$ competitors run in order to reach on the arrival line $L$. Each runner has his special sign (on the shirt): $A, B, C, D$ etc.

Work hypothesis 1. We suppose [1] that more then one runner could ocuppy the same place. We denote by $t_{A 1}$ the time for the runner $A$ and $t_{B 1}$ the time for $B$.

If $\left|t_{A 1}-t_{B 1}\right| \in[0, \varepsilon 1]$, where $\varepsilon 1>0$ is very small (in millisecomds), then both runners will ocupy the same Place1= I.

If $\left|t_{A 2}-t_{B 2}\right| \in[0, \varepsilon 2]$, where $\varepsilon 2>0$ and $\varepsilon 2<\varepsilon 1$ is very small (in milli-secomds), then both runners will ocupy the same Place2=II etc.

The total number of runners arriving on a special (particular) place is denoted by

I II III IV etc

$$
\begin{gathered}
i \quad j \quad k \quad l \cdots \cdots \cdots \cdots p ; i \neq 0, \text { where } \\
i, j, k, l, \cdots, p \in\{0,1,2,3,4, \cdots, n\} \\
i+j+k+l+\cdots+p=n .
\end{gathered}
$$$$
\text { and }
$$

Definition 1. The set $(i, j, k, \cdots, p)$ is called non-nominal state. By the value $i$ is known only the total total number of runners (not their names) ocuppying the place $\mathrm{I}$; by $j$ is known only the total number of runners ocuppying the place II etc.

The value $N=N(n)$ is the total number of non-nominal 
states. The question is to find the formula for $N(n)$.

Example 1. $n=5,(3,1,1,0,0),(2,2,1,0,0)$ are nonnominal states. The set $(3,1,0,1,0)$ is not a correctly nonnominal state.

Proposition 1 . The total number of non-nominal states $[1,3]$ is

$$
N=N(n)=2^{n-1}, n \geq 1
$$

Proof. We use the equation $i+j+k+l+\cdots+p=n$. This equation has only the solutions in natural numbers. The whole number of solutions is $N(n)=2^{n-1}$. End.

\section{Examples of Non-nominal States and Verify the Total Number $N=N(n)=2^{n-1}$}

Each runner could ocuppay (on the podium, in decreasing order of importance) the places I or II or III etc. Thus, Place $=I$, Place $2=I I$, Place $3=I I I$ etc are the positions on the arival line; PlaceX $\in\{1,2, \cdots, n\}$.

Application 1. We ilustrare all the non-nominal states for several value of $n$ are:

\begin{tabular}{|c|c|c|c|c|c|}
\hline$n=1$ & $n=2$ & $n=3$ & $n=4$ & $n=5$ & \\
\hline I & I II & I II III & I II III IV & I II III IV V & $s$ \\
\hline (1) & $(2,0)$ & $(3,0,0)$ & $(4,0,0,0)$ & $(5,0,0,0,0)$ & $s=1$ \\
\hline \multirow[t]{16}{*}{$N=1$} & $(1,1)$ & $(2,1,0)$ & $(3,1,00)$ & $(4,1,0,0,0)$ & $s=2$ \\
\hline & $N=2$ & $(1,2,0)$ & $(2,2,0,0)$ & $(3,2,0,0,0)$ & $s=3$ \\
\hline & & $(1,1,1)$ & $(2,1,1,0)$ & $(3,1,1,0,0)$ & $s=4$ \\
\hline & & $N=4$ & $(1,3,0,0)$ & $(2,3,0,0,0)$ & $s=5$ \\
\hline & & & $(1,2,1,0)$ & $(2,2,1,0,0)$ & $s=6$ \\
\hline & & & $(1,1,2,0)$ & $(2,1,2,0,0)$ & $s=7$ \\
\hline & & & $(1,1,1,1)$ & $(2,1,1,1,0)$ & $s=8$ \\
\hline & & & $N=8$ & $(1,4,0,0,0)$ & $s=9$ \\
\hline & & & & $(1,3,1,0,0)$ & $s=10$ \\
\hline & & & & $(1,2,2,0,0)$ & $s=11$ \\
\hline & & & & $(1,2,1,1,0)$ & $s=12$ \\
\hline & & & & $(1,1,3,0,0)$ & $s=13$ \\
\hline & & & & $(1,1,2,1,0)$ & $s=14$ \\
\hline & & & & $(1,1,1,2,0)$ & $s=15$ \\
\hline & & & & $(1,1,1,1,1)$ & $s=16$ \\
\hline & & & & $N=16$ & \\
\hline
\end{tabular}

Observation 1. At the consrtuction of all non-nominal states one recommends $[1,4]$ that the string for the position I to use a decreasing string. The same rule is validly for position II, but for sub-strings etc.

We count the non-nominl states by the variable $s$ and $s=1, N(n)$.

Each non-nominal state $s$ generates the nominal states, where on indicates the name of each runner.

$n=4,(A B, C, D,-),(A, B C, D,-),(B, C, A D,-)$ are examples of nominal states.

New notations. $T=T(n ; s)$ is the total number of nominal states generated by the non-nominal state number $s, 1 \leq s \leq N(n)$.

$S(n)=$ is the total number of nominal states for a given $n$.

There exists the relation

$$
S(n)=\sum_{s=1}^{N} T(n ; s)
$$

It remains the problem of computing the value $T(n ; s)$, for $1 \leq s \leq N$.

Proposition 2. [5] The total number of nominal states, related with $n$ and $1 \leq s \leq N$, illustrated by several particular cases is

$$
\begin{gathered}
s,(i, j, k, l), i+j+k+l=4, T(n ; s)=C_{n}^{i} C_{n-i}^{j} C_{n-i-j}^{k} C_{n-i-j-k}^{l} \\
n=4, N(4)=8, s=6,(i, j, k, l)=(1,2,1,0), i+j+k+l=4 . \\
T(4 ; 6)=C_{n}^{i} C_{n-i}^{j} C_{n-i-j}^{k}=C_{4}^{1} C_{4-1}^{2} C_{4-1-2}^{1}=C_{4}^{1} C_{3}^{2} C_{1}^{1}=4 \cdot 3 \cdot 1=12 . \\
n=5, N(5)=16, s=12,(i, j, k, l, p)=(1,2,1,1,0), i+j+k+l=5 \\
T(5 ; 12)=C_{n}^{i} C_{n-i}^{j} C_{n-i-j}^{k} C_{n-i-j-k}^{l}=C_{5}^{1} C_{5-1}^{2} C_{5-1-2}^{1} C_{5-1-2-1}^{1}=C_{5}^{1} C_{4}^{2} C_{2}^{1} C_{1}^{1}=60 .
\end{gathered}
$$

The generalisation for any $n$ and $s$ is immediately.

Observation 2. The total number of nominal states $S(n)$ could be calculated by two methods.

Method 1. For a small value of $n$ we construct the whole 
set of nominal states and (directly) count the total number $S(n)$.

Method 2. Generate all $s=1, N(n)$ non-nominal states $(i, j, k, \cdots)$. Generate all nominal states. Use formula (3) for $T=T(n ; s)$ and (2) for $S(n)$

\section{The Algorithm of Arrival Line}

Step 1. Let $n$ be a given natural number, $n \geq 1$.

Calculate the total number $N=N(n)=2^{n-1}$ of all nonnominal states.

Step 2. Construct the set of all non-nominal states $(i, j, k, \cdots, p)$, for $s=1, N$.

Step 3. For each non-nominal state $s, 1 \leq s \leq N$ we construct the set of all nominal states. Each state has $T(n ; s)$ nominal states, where each runner is known by its identidication sign or number.

Step 4. For each non-nominal state $s$ calculate the natural number of all nominal states $T(n ; s), s=1, N$.

Step 5. For given value $n$ calculate the total number of nominal states

$$
S(n)=\sum_{s=1}^{N} T(n ; s)
$$

Step 6. Calculate partial frequency and final frequency for nominale states.

(see section 5).

\section{Numerical Examples for $\boldsymbol{n}=\mathbf{4}$ and $\boldsymbol{n}=\mathbf{5}$}

Application 2. For $n=4$ the runners are $A, B, C, D$.

Step 1. $N=N(4)=8$ non-nominal states $(i, j, k, l)$

Step 2. $i, j, k, l \in\{0,1,2,3,4\}, i+j+k+l=4$

See application 1.

\begin{tabular}{ll}
\hline$s=1$ & $(4,0,0,0)$ \\
$s=2$ & $(3,1,0,0)$ \\
$s=3$ & $(2,2,0,0)$ \\
$s=4$ & $(2,1,1,0)$ \\
$s=5$ & $(1,3,0,0)$ \\
$s=6$ & $(1,2,1,0)$ \\
$s=7$ & $(1,1,2,0)$ \\
$s=8$ & $(1,1,1,1)$ \\
\hline
\end{tabular}

Step 3. For each $s$ we construct the set of all nominal states.

\begin{tabular}{|c|c|c|c|c|c|c|c|}
\hline Place1 & Place2 & Place3 & Place4 & Place1 & Place2 & Place3 & Place4 \\
\hline$s=1$ & $(4,0,0,0)$ & $s=5$ & $(1,3,0,0)$ & & & & \\
\hline $\mathrm{ABCD}$ & - & - & - & A & BCD & - & - \\
\hline$s=2$ & & $(3,1,0,0)$ & B & ACD & - & - & \\
\hline $\mathrm{ABC}$ & $\mathrm{D}$ & - & - & $\mathrm{C}$ & $\mathrm{ABD}$ & - & - \\
\hline $\mathrm{ABD}$ & $\mathrm{C}$ & - & - & $\mathrm{D}$ & $\mathrm{ABC}$ & - & - \\
\hline $\mathrm{ACD}$ & B & - & - & $s=6$ & $(1,2,1,0)$ & & \\
\hline $\mathrm{BCD}$ & A & - & - & A & $\mathrm{BC}$ & $\mathrm{D}$ & -1 \\
\hline$s=3$ & & $(2,2,0,0)$ & A & $\mathrm{BD}$ & $\mathrm{C}$ & -2 & \\
\hline $\mathrm{AB}$ & $\mathrm{CD}$ & - & - & A & $\mathrm{CD}$ & B & -3 \\
\hline $\mathrm{AC}$ & $\mathrm{BD}$ & - & - & B & $\mathrm{AC}$ & $\mathrm{D}$ & -4 \\
\hline $\mathrm{AD}$ & $\mathrm{BC}$ & - & - & B & $\mathrm{AD}$ & $\mathrm{C}$ & -5 \\
\hline $\mathrm{BC}$ & $\mathrm{AD}$ & - & - & B & $\mathrm{CD}$ & A & -6 \\
\hline $\mathrm{BD}$ & $\mathrm{AC}$ & - & - & $\mathrm{C}$ & $\mathrm{AB}$ & $\mathrm{D}$ & -7 \\
\hline $\mathrm{CD}$ & $\mathrm{AB}$ & - & - & $\mathrm{C}$ & $\mathrm{AD}$ & B & -8 \\
\hline$s=4$ & $(2,1,1,0)$ & $\mathrm{C}$ & $\mathrm{BD}$ & A & -9 & & \\
\hline $\mathrm{AB}$ & $\mathrm{C}$ & $\mathrm{D}$ & - & $\mathrm{D}$ & $\mathrm{AB}$ & $\mathrm{C}$ & -10 \\
\hline $\mathrm{AB}$ & $\mathrm{D}$ & $\mathrm{C}$ & - & $\mathrm{D}$ & $\mathrm{AC}$ & B & -11 \\
\hline $\mathrm{AC}$ & B & $\mathrm{D}$ & - & $\mathrm{D}$ & $\mathrm{BC}$ & A & -12 \\
\hline $\mathrm{AC}$ & $\mathrm{D}$ & B & - & \multicolumn{4}{|c|}{ Verification $s=6 ; 12$} \\
\hline $\mathrm{AD}$ & B & $\mathrm{C}$ & - & & & & \\
\hline $\mathrm{AD}$ & $\mathrm{C}$ & B & - & & & & \\
\hline $\mathrm{BC}$ & A & $\mathrm{D}$ & - & & & & \\
\hline $\mathrm{BC}$ & $\mathrm{D}$ & A & - & & & & \\
\hline $\mathrm{BD}$ & A & $\mathrm{C}$ & - & & & & \\
\hline $\mathrm{BD}$ & $\mathrm{C}$ & A & - & & & & \\
\hline CD & A & B & - & & & & \\
\hline CD & B & A & - & & & & \\
\hline$s=7$ & $(1,1,2,0)$ & $s=8$ & $(1,1,1,1)$ & & & & \\
\hline A & B & $\mathrm{CD}$ & - & B & $\mathrm{C}$ & $\mathrm{D}$ & A \\
\hline A & $\mathrm{C}$ & BD & - & B & $\mathrm{C}$ & A & D \\
\hline A & D & $\mathrm{BC}$ & - & B & D & $\mathrm{C}$ & A \\
\hline B & A & $\mathrm{CD}$ & - & B & D & A & $\mathrm{C}$ \\
\hline B & $\mathrm{C}$ & $\mathrm{AD}$ & - & B & A & $\mathrm{C}$ & D \\
\hline B & D & $\mathrm{AC}$ & - & B & A & $\mathrm{D}$ & $\mathrm{C}$ \\
\hline$s=7$ & $(1,1,2,0)$ & $s=8$ & $(1,1,1,1)$ & & & & \\
\hline $\mathrm{C}$ & D & $\mathrm{AB}$ & - & $\mathrm{C}$ & D & A & B \\
\hline $\mathrm{C}$ & B & $\mathrm{AD}$ & - & $\mathrm{C}$ & D & B & A \\
\hline $\mathrm{C}$ & A & $\mathrm{BD}$ & - & $\mathrm{C}$ & A & $\mathrm{D}$ & B \\
\hline D & A & $\mathrm{BC}$ & - & $\mathrm{C}$ & A & B & D \\
\hline $\mathrm{D}$ & B & $\mathrm{AC}$ & - & $\mathrm{C}$ & B & D & A \\
\hline
\end{tabular}




\begin{tabular}{llllllll}
\hline Place1 & Place2 & Place3 & Place4 & Place1 & Place2 & Place3 & Place4 \\
\hline D & C & AB & - & C & B & A & D \\
& D & A & B & C & C & & \\
A & $(1,1,1,1)$ & D & A & D & D & A & C \\
A & B & C & D & D & B & C & A \\
A & B & D & C & C & A & B \\
A & C & B & D & D & B & A \\
A & C & D & B & D & & \\
A & D & B & C & Verification s $=8 ; 24$ & & \\
\hline
\end{tabular}

Step 4. For each non-nominal state $s$ calculate the natural number of all nominal states $T(4 ; s), s=1,8$.

$$
\begin{gathered}
T(4 ; s),(i, j, k, l), i+j+k+l=4, \\
T(n ; s)=C_{n}^{i} C_{n-i}^{j} C_{n-i-j}^{k} C_{n-i-j-k}^{l} \\
s=1,(4,0,0,0), T(4 ; 1)=C_{4}^{4}=1 \\
s=2,(3,1,0,0), T(4 ; 2)=C_{4}^{3} C_{4-3}^{1}=4 \\
s=3,(2,2,0,0), T(4 ; 3)=C_{4}^{2} C_{4-2}^{2}=6 \\
s=4,(2,1,1,0), T(4 ; 4)=C_{4}^{2} C_{4-2}^{1} C_{4-2-1}^{1}=12 \\
s=5,(1,3,0,0), T(4 ; 5)=C_{4}^{1} C_{4-1}^{3}=4 \\
s=6,(1,2,1,0), T(4 ; 6)=C_{4}^{1} C_{4-1}^{2} C_{4-1-2}^{1}=12 \\
s=7,(1,1,2,0), T(4 ; 7)=C_{4}^{1} C_{4-1}^{1} C_{4-1-1}^{2}=12 \\
s=8,(1,1,1,1), T(4 ; 8)=C_{4}^{1} C_{4-1}^{1} C_{4-1-1}^{1} C_{4-1-1-1}^{1} 24
\end{gathered}
$$

\begin{tabular}{|c|c|c|c|c|c|c|c|c|c|}
\hline Place1 & Place2 & Place3 & Place4 & Place5 & Place1 & Place2 & Place3 & Place4 & Place5 \\
\hline$s=1$ & $(5,0,0,0,0)$ & $s=4$ & $(3,1,1,0,0)$ & & & & & & \\
\hline $\mathrm{ABCDE}$ & - & - & - & - & $\mathrm{ABC}$ & D & E & - & - \\
\hline$s=2$ & $(4,1,0,0,0)$ & $\mathrm{ABC}$ & E & D & - & - & & & \\
\hline $\mathrm{ABCD}$ & E & - & - & - & $\mathrm{ABD}$ & $\mathrm{C}$ & E & - & - \\
\hline $\mathrm{ABCE}$ & D & - & - & - & $\mathrm{ABD}$ & E & $\mathrm{C}$ & - & - \\
\hline ABDE & C & - & - & - & $\mathrm{ABE}$ & $\mathrm{C}$ & D & - & - \\
\hline ACDE & B & - & - & - & $\mathrm{ABE}$ & D & $\mathrm{C}$ & - & - \\
\hline BCDE & A & - & - & - & ACD & B & E & - & - \\
\hline$s=3$ & $(3,2,0,0,0)$ & ACD & E & B & - & - & & & \\
\hline $\mathrm{ABC}$ & $\mathrm{DE}$ & - & - & - & ACE & B & $\mathrm{D}$ & - & - \\
\hline $\mathrm{ABD}$ & $\mathrm{CE}$ & - & - & - & ACE & D & B & - & - \\
\hline $\mathrm{ABE}$ & $\mathrm{CD}$ & - & - & - & $\mathrm{ADE}$ & B & $\mathrm{C}$ & - & - \\
\hline ACD & $\mathrm{BE}$ & - & - & - & $\mathrm{ADE}$ & $\mathrm{C}$ & B & - & - \\
\hline $\mathrm{ACE}$ & $\mathrm{BD}$ & - & - & - & $\mathrm{BCD}$ & A & E & - & - \\
\hline $\mathrm{ADE}$ & $\mathrm{BC}$ & - & - & - & BCD & E & A & - & - \\
\hline BCD & $\mathrm{AE}$ & - & - & - & BCE & A & D & - & - \\
\hline BCE & $\mathrm{AD}$ & - & - & - & BCE & D & A & - & - \\
\hline BDE & $\mathrm{AC}$ & - & - & - & $\mathrm{BDE}$ & A & $\mathrm{C}$ & - & - \\
\hline CDE & $\mathrm{AB}$ & - & - & - & BDE & $\mathrm{C}$ & A & - & - \\
\hline $\mathrm{CDE}$ & A & B & - & - & & & & & \\
\hline $\mathrm{CDE}$ & B & A & - & - & & & & & \\
\hline$s=5$ & $(2,3,0,0,0)$ & & & & & & & & \\
\hline $\mathrm{AB}$ & $\mathrm{CDE}$ & - & - & - & BD & ACE & - & - & - \\
\hline $\mathrm{AC}$ & BDE & - & - & - & $\mathrm{BE}$ & ACD & - & - & - \\
\hline $\mathrm{AD}$ & BCE & - & - & - & $\mathrm{CD}$ & $\mathrm{ABE}$ & - & - & - \\
\hline $\mathrm{AE}$ & $\mathrm{BCD}$ & - & - & - & $\mathrm{CE}$ & $\mathrm{ABD}$ & - & - & - \\
\hline
\end{tabular}

Step 5. Calculate the total number of nominal states $S(n)=\sum_{s=1}^{N} T(n ; s)$.
$S(4)=\sum_{s=1}^{8} T(4 ; s)=1+4+\ldots \ldots+24=75$ nominal states.

Application 3. For $n=5$ the runners are $A, B, C, D, E$.

Step 1. $N=N(5)=16$ non-nominal states $(i, j, k, l, p)$

Step 2. $i, j, k, l, p \in\{0,1,2,3,4,5\}, i+j+k+l+p=5$

See application 1 .

\begin{tabular}{ll}
\hline$s=1$ & $(5,0,0,0,0)$ \\
$s=2$ & $(4,1,0,0,0)$ \\
$s=3$ & $(3,2,0,0,0)$ \\
$s=4$ & $(3,1,1,0,0)$ \\
$s=5$ & $(2,3,0,0,0)$ \\
$s=6$ & $(2,2,1,0,0)$ \\
$s=7$ & $(2,1,2,0,0)$ \\
$s=8$ & $(2,1,1,1,0)$ \\
$s=9$ & $(1,4,0,0,0)$ \\
$s=10$ & $(1,3,1,0,0)$ \\
$s=11$ & $(1,2,2,0,0)$ \\
$s=12$ & $(1,2,1,1,0)$ \\
$s=13$ & $(1,1,3,0,0)$ \\
$s=14$ & $(1,1,2,1,0)$ \\
$s=15$ & $(1,1,1,2,0)$ \\
$s=16$ & $(1,1,1,1,1)$ \\
\hline
\end{tabular}

Step 3. For each $s$ we construct the set of all nominal states. 


\begin{tabular}{llllllllll}
\hline Place1 & Place2 & Place3 & Place4 & Place5 & Place1 & Place2 & Place3 & Place4 & Place5 \\
\hline $\mathrm{BC}$ & $\mathrm{ADE}$ & - & - & - & $\mathrm{DE}$ & $\mathrm{ABC}$ & - & - & - \\
\hline
\end{tabular}

Observation 3. For the others values of $s$ exists a big number of nominal states. That is why we simplify their representation.

$s=6 \quad(2,2,1,0,0),($ Place, Placec2, Place3, Place4,Place5)

$(\mathrm{AB}, \mathrm{CD}, \mathrm{E},-,-)$,

(AC, BD, E, -, -),

(AD, BC, E, -, -),

(AE, BC, D, -, -),

(BC, AD, E, -, -),

(BD, AC, E, -, -),

(BE, AC, D, -, -),

(CD, AB, E, -, -),

(CE, AB, D, - , -),

(DE, AC, B, -, -),

$s=7 \quad(2,1,2,0,0)$

(AB, E, CD, -, -),

(AC, E, BD, -, -),

(AD, E, BC, -, -),

(AE, D, BC, -, -),

$(\mathrm{BC}, \mathrm{E}, \mathrm{AD},-,-)$,

(BD, E, AC, -, -),

(BE, D, AC, -, -),

(CD, E, AB, -, -),

(CE, D, AB, -, -),

(DE, B, AC, -, -),
(AB, CE, D, -, -),

(AC, BE, D, -, -),

(AD, BE, C, -, -),

(AE, BD, C, -, -),

(BC, AE, D, -, -),

(BD, AE, C, -, -),

(BE, AD, C, -, -),

(CD, AE, B, -, -),

(CE, AD, B, -, -),

(DE, AB, C, -, -),

, (Place1, Placec2, Place3, Place4, Place5)

(AB, D, CE, -, -),

(AC, D, BE, -, -),

(AD, C, BE, -, -),

(AE, C, BD, -, -),

(BC, D, AE, -, -),

(BD, C, AE, -, -),

(BE, C, AD, -, -),

(CD, B, AE, -, -),

(CE, B, AD, -, -),

(DE, C, AB, -, -),
(AB, DE, C, -, -)

(AC, DE, B, -, -)

(AD, CE, B, -, -)

(AE, CD, B, -, -)

(BC, DE, A, -, -)

(BD, CE, A, -, -)

(BE, CD, A, -, -)

(CD, BE, A, -, -)

(CE, BD, A, -, -)

(DE, BC, A, -, -)

(AB, C, DE, -, -)

(AC, B, DE, -, -)

(AD, B, CE, -, -)

(AE, B, CD, -, -)

(BC, A, DE, -, -)

(BD, A, CE, -, -)

(BE, A, CD, -, -)

(CD, A, BE, -, -)

(CE, A, BD, -, -)

(DE, A, BC, -, -) $s=8(2,1,1,1,0)$

$(\mathrm{AB}, \mathrm{C}, \mathrm{D}, \mathrm{E},-),(\mathrm{AB}, \mathrm{C}, \mathrm{E}, \mathrm{D},-),(\mathrm{AB}, \mathrm{D}, \mathrm{C}, \mathrm{E},-)$ and so on.

$s=9(1,4,0,0,0)$

(A, BCDE, -, -, -), (B, ACDE, -, -, -), (C, ABDE, -, -, -) and so on.

Step 4. For each non-nominal state $s$ calculate the natural number of all nominal states $T(5 ; s), s=1,16$.

$$
\begin{gathered}
T(n ; s)=C_{n}^{i} C_{n-i}^{j} C_{n-i-j}^{k} C_{n-i-j-k}^{l} C_{n-i-j-k-l}^{p}, 1 \leq s \leq N=16 \\
S(n)=\sum_{s=1}^{N} T(n ; s), N=N(5)=16, \\
n=5,(i, j, k, l, p), i+j+k+l+p=5 . \\
T(n ; s)=C_{n}^{i} C_{n-i}^{j} C_{n-i-j}^{k} C_{n-i-j-k}^{l} C_{n-i-j-k-l}^{p}, 1 \leq s \leq N
\end{gathered}
$$

$T(5 ; 1)=C_{5}^{5}=1 \quad T(5 ; 2)=C_{5}^{4} C_{1}^{1}=5 \quad T(5 ; 3)=10$

$T(5 ; 4)=20 \quad T(5 ; 5)=10 \quad T(5 ; 6)=30$

$T(5 ; 7)=30 \quad T(5 ; 8)=60 \quad T(5 ; 9)=5$

$T(5 ; 10)=20 \quad T(5 ; 11)=60 \quad T(5 ; 12)=20$

$T(5 ; 13)=30 \quad T(5 ; 14)=60 \quad T(5 ; 15)=60$

$T(5 ; 16)=5 !=120$.

$S(5)=\sum_{s=1}^{16} T(5 ; s)=541$ nominal states.

\section{Partial Frequency and Final Frequency for Nominal States}

Computation formulas

The notions partial frequency and final frequency [6] are defined for a nominal known runner in final classification.

Definitions:

$P F(n ; s ;$ Runner, PlaceX $)$ is the partial frequency $=$ the number of favorable cases for a nominated runner A or B or $\mathrm{C}$ etc. to occupy the position PlaceX in the final classification, for the non-nominal state $s, 1 \leq s \leq N(n)$.

$F F(n ;$ Runner, Place $X)$ is the final frequency $=$ the number of favorable cases for a nominated runner $\mathrm{A}$ or $\mathrm{B}$ or $\mathrm{C}$ etc. to occupy the position PlaceX in the final classification.

Example 2. $P F(n=4 ; 3 ; A ; I), \quad P F(n=5 ; 4 ; A ; I)$, $P F(n=5 ; 7 ; B ; I I)$.

$$
F F(n=4 ; A ; I), F F(n=5 ; A ; I), F F(n=5 ; B ; I I) \text {. }
$$

In an informatics model, the values $F P$ and $F F$ could be used as the functions having 4 or 3 variables, respectively.

Proposition 3. The partial frequency $P F(n ; s ;$ Runner, PlaceX) computing formula is justified and illustrated for any $n \geq 2$ and for some particular $s$,

$$
1 \leq s \leq N \text {. }
$$

$n, s,(i, j, k, l), i+j+k+l=n$, runner A, place $I$

$n-1, s I,(i-1, j, k, l),(i-1)+j+k+l=n-1$, runner A, place $I$

$$
\begin{gathered}
P F(n ; s ; A ; I)=C_{n-1}^{i-1} C_{n-1-(i-1)}^{j} C_{n-1-(i-1)-j}^{k} C_{n-1-(i-1)-j-k}^{l} \text { (5) } \\
P F(n ; s ; A ; I)=C_{n-1}^{i-1} C_{n-i}^{j} C_{n-i-j}^{k} C_{n-i-j-k}^{l} \\
n, s,(i, j, k, l), i+j+k+l=n, \text { runner A, place } I I \\
n-1, s I I,(i, j-1, k, l), i+(j-1)+k+l=n-1, \text { runner }
\end{gathered}
$$

A, place $I I$ 


$$
\begin{gathered}
P F(n ; s ; A ; I I)=C_{n-1}^{i} C_{n-1-i}^{j-1} C_{n-1-i-(j-1)}^{k} C_{n-1-i-(j-1)-k}^{l}(7) \\
P F(n ; s ; A ; I I)=C_{n-1}^{i} C_{n-1-i}^{j-1} C_{n-i-j}^{k} C_{n-i-j-k}^{l} \\
n, s,(i, j, k, l), i+j+k+l=n, \text { runner A, place } I I I \\
n-1, s I I I,(i, j, k-1, l), i+j+(k-1)+l=n-1, \text { runner }
\end{gathered}
$$
A, place $I I I$

$$
\begin{gathered}
P F(n ; s ; A ; I I I)=C_{n-1}^{i} C_{n-1-i}^{j} C_{n-1-i-j}^{k-1} C_{n-1-i-j-(k-1)}^{l}(9) \\
P F(n ; s ; A ; I I I)=C_{n-1}^{i} C_{n-1-i}^{j} C_{n-1-i-j}^{k-1} C_{n-i-j-k}^{l} \text { (10) } \\
n, s,(i, j, k, l), i+j+k+l=n, \text { runner A, place } I V \\
n-1, s I V,(i, j, k, l-1), i+j+k+(l-1)=n-1, \text { runner }
\end{gathered}
$$
A, place $I V$

$$
\begin{aligned}
& P F(n ; s ; A ; I V)=C_{n-1}^{i} C_{n-1-i}^{j} C_{n-1-i-j}^{k} C_{n-1-i-j-k}^{l-1} \\
& P F(n ; s ; A ; I V)=C_{n-1}^{i} C_{n-1-i}^{j} C_{n-1-i-j}^{k} C_{n-1-i-j-k}^{l-1}
\end{aligned}
$$

For final frequency $F F(n ;$ Runner, PlaceX $)$ one uses the formula

$$
F F(n ; \text { Runner }, \text { PlaceX })=\sum_{s=1}^{N} P F(n ; s ; \text { Runner } ; \text { PlaceX })
$$

Observation 4. For non-nominal states having particular forms, like $(i, j, 0.0,0)$ or $(i, i, 0.0,0)$ etc we can use some speculative methods based on difference method or proportional method. But, the reccomended formulas are (5), (7), (9), (11).

Application 4. $n=4, s=3,(2,2,0,0), 2+2+0+0=4$. $n-1=3, s I,(1,2,0,0), 1+2+0+0=3$, runner A, place $I$

$$
P F(4 ; 3 ; A ; I)=C_{3}^{1} C_{3-1}^{2}=3
$$

$n-1=3, s I I,(2,1,0,0), 2+1+0+0=3$, runner A, place II

$P F(4 ; 3 ; A ; I I)=C_{3}^{2} C_{3-2}^{1}=3$. Stop for $s=3$, because $k=l=0$

$$
P F(4 ; 3 ; A ; I I I)=0, P F(4 ; 3 ; A ; I V)=0 .
$$

Application 5. $n=4, s=8,(1,1,1,1), 1+1+1+1=4$. $n-1=3, s I,(0,1,1,1), 0+1+1+1=3$, runner A, place I

$$
P F(4 ; 8 ; A ; I)=C_{3}^{0} C_{3-0}^{1} C_{3-1}^{1} C_{3-1-1}^{1}=6
$$

$n-1=3$, sII $,(1,0,1,1), 1+0+1+1=3$, runner A, place $I I$

$$
P F(4 ; 8 ; A ; I I)=C_{3}^{1} C_{3-1}^{0} C_{3-1}^{1} C_{3-1-1}^{1}=6
$$

$n-1=3$, sIII $,(1,1,0,1), 1+1+0+1=3$, runner A, place
III

$$
P F(4 ; 8 ; A ; I I)=C_{3}^{1} C_{3-1}^{1} C_{3-1-1}^{0} C_{3-1-1}^{1}=6
$$

$n-1=3, s I V,(1,1,1,0), 1+1+1+0=3$, runner A, place IV

$$
P F\left(4 ; 8 ; A ; I V=C_{3}^{1} C_{3-1}^{1} C_{3-1-1}^{1} C_{3-1-1-1}^{0}=6 .\right.
$$

Application 6. Calculate $P F$ ) and $F F$. Correctitude verification.

$n=3 ; A, B, C ; N(3)=4 ; s=1,4 ; S(3)=1+3+3+6=13$

$P F(3 ; 1 ; A ; I)=1, P F(3 ; 2 ; A ; I)=2, P F(3 ; 3 ; A ; I)=1$

$P F(3 ; 4 ; A ; I)=2$ and $F F(3 ; A ; I)=1+2+1+2=6$

$$
\begin{gathered}
P F(3 ; 1 ; A ; I I)=0, P F(3 ; 2 ; A ; I I)=1, \\
P F(3 ; 3 ; A ; I I)=2
\end{gathered}
$$

$P F(3 ; 4 ; A ; I I)=2$ and $F F(3 ; A ; I I)=1+2+1+2=5$

$P F(3 ; 1 ; A ; I I I)=0, P F(3 ; 2 ; A ; I I I)=0$,

$$
P F(3 ; 3 ; A ; I I I)=0
$$

$P F(3 ; 4 ; A ; I I I)=2$ and $F F(3 ; A ; I I I)=0+0+0+2=2$

Correctitude verification

$F F(3 ; A ; I)+F F(3 ; A ; I I)+$

$F F(3 ; A ; I I I)=6+5+2=13=S(3)$. True

Application 7. Calculate $P F$ and $F F$. Correctitude verfication.

$$
n=4 ; A, B, C, D ; N(4)=8 ; s=1,8 ; S(4)=75
$$

We orgonize the results in an adequate table 1.

Table 1. PF FF $n=4$.

\begin{tabular}{lllll}
\hline PFI & PFII & PFIII & PFIV & \\
\hline $\mathrm{s}=1$ & 1 & 0 & 0 & 0 \\
$\mathrm{~s}=2$ & 3 & 1 & 0 & 0 \\
$\mathrm{~s}=3$ & 3 & 3 & 0 & 0 \\
$\mathrm{~s}=4$ & 6 & 3 & 3 & 0 \\
$\mathrm{~s}=5$ & 1 & 3 & 0 & 0 \\
$\mathrm{~s}=6$ & 3 & 6 & 3 & 0 \\
$\mathrm{~s}=7$ & 3 & 3 & 6 & 0 \\
$\mathrm{~s}=8$ & 6 & 6 & 6 & 6 \\
$F F$ & 26 & 25 & 18 & 6 \\
\hline
\end{tabular}

Verification. $26+25+18+6=75, S(n=4)=75$. True.

Application 8. Calculate $P F$ and FF . Correctitude verfication.

$n=5 ; A, B, C, D, E ; N(5)=16 ; s=1,16 ; S(5)=541$

We orgonize the results in an adequate table 2. 
Table 2. PF FF $n=5$.

\begin{tabular}{llllll}
\hline PFI & PFII & PFIII & PFIV & PFV & \\
\hline $\mathrm{s}=1$ & 1 & 0 & 0 & 0 & 0 \\
$\mathrm{~s}=2$ & 4 & 1 & 0 & 0 & 0 \\
$\mathrm{~s}=3$ & 6 & 4 & 0 & 0 & 0 \\
$\mathrm{~s}=4$ & 12 & 4 & 4 & 0 & 0 \\
$\mathrm{~s}=5$ & 4 & 6 & 0 & 0 & 0 \\
$\mathrm{~s}=6$ & 12 & 12 & 6 & 0 & 0 \\
$\mathrm{~s}=7$ & 12 & 6 & 12 & 0 & 0 \\
$\mathrm{~s}=8$ & 24 & 12 & 12 & 12 & 0 \\
$\mathrm{~s}=9$ & 1 & 4 & 0 & 0 & 0 \\
$\mathrm{~s}=10$ & 4 & 12 & 4 & 0 & 0 \\
$\mathrm{~s}=11$ & 6 & 12 & 12 & 0 & 0 \\
$\mathrm{~s}=12$ & 12 & 24 & 12 & 12 & 0 \\
$\mathrm{~s}=13$ & 4 & 4 & 12 & 0 & 0 \\
$\mathrm{~s}=14$ & 12 & 12 & 24 & 12 & 0 \\
$\mathrm{~s}=15$ & 12 & 12 & 12 & 24 & 0 \\
$\mathrm{~s}=16$ & 24 & 24 & 24 & 24 & 24 \\
$F F$ & 150 & 149 & 134 & 84 & 24 \\
\hline
\end{tabular}

Verification. $150+149+134+84+24=541, S(n=5)=541$. True.

\section{The Random Variables Attached to Final Classification}

The probability of each place on arrival line [2,7]

Let $n$ be the runners $A, B, C, D, E, F$ etc reaching on arrival line.

We know the total number of nominal states $S(n)$ and the total number of favorable cases $F F(n$; Runner, PlaceX) for each runner in final classification. We simplify the writing and denote $F F(n ;$ Name, Place $)=F F n I$, $F F(n ;$ Name, Place 2$)=F F n I I$ $F F(n ;$ Name, Place $)=F F n$ III etc.

The occupied places could be Place 1, Place 2, .., Placen.

Observation. May be the runerr is intersted

a) to know the probability to ocuppay place I or

a) to know the probability to obtain the number of $\alpha=100$ points.

The score rule. Each runner occupiyng the position PlaceX receives a score (a number of points). The score is at choice of organizers.

For Placel the value of score is $\alpha$ points. For example $\alpha=100$ points.

For Place 2 the value of score is $\alpha / 2$ points or $2 \alpha / 3$ points etc.

For Place 3 the value of score is $\alpha / 3$ points or $2 \alpha / 4$ points etc.

A discrete random variable in a classical representation has the form

$$
\alpha=100
$$

$$
\begin{gathered}
X=\left(\begin{array}{ccc}
x_{1} & x_{2} & x_{3} \cdots \cdots x_{n} \\
p_{1} & p_{2} & p_{3} \cdots \cdots p_{n}
\end{array}\right), 0 \leq p_{i} \leq 1, \\
p_{1}+p_{2}+\cdots+p_{n}=1
\end{gathered}
$$

For the arrival line we mention several random variables

$$
\begin{gathered}
X(3)=\left(\begin{array}{cccc}
I & I I & I I I \\
\frac{F F 3 I}{S(3)} & \frac{F F 3 I I}{S(3)} & \frac{F F 3 I I I}{S(3)}
\end{array}\right) \\
X(4)=\left(\begin{array}{ccccc}
I & I I & I I I & I V \\
\frac{F F 4 I}{S(4)} & \frac{F F 4 I I}{S(4)} & \frac{F F 4 I I I}{S(4)} & \frac{F F 4 I V}{S(4)}
\end{array}\right) \\
X(5)=\left(\begin{array}{ccccc}
I & I I & I I I & I V & V \\
\frac{F F 5 I}{S(5)} & \frac{F F 5 I I}{S(5)} & \frac{F F 5 I I I}{S(5)} & \frac{F F 5 I V}{S(5)} & \frac{F F 5 V}{S(5)}
\end{array}\right) .
\end{gathered}
$$

If we use the random variable in relation with the score rule, with $\alpha=100$ points, then we obtain

$$
X(n)=\left(\begin{array}{cccc}
\frac{100}{1} & \frac{100}{2} & \frac{100}{3} \cdots \cdots \cdot \frac{100}{n} \\
\frac{F F n I}{S(n)} & \frac{F F n I I}{S(n)} & \frac{F F n I I I}{S(n)} \cdots \frac{F F n n}{S(n)}
\end{array}\right)
$$

where

$F F n I+F F n I I+\cdots+F F n n=S(n)$, for any natural number $n \geq 1$.

The random variable $X(n)$ defines the random variation of score, on arrival line. This random variable gives the possibility to execute a lot of statistical compuations $[8,9]$ like:

mean value $M) X)=m$, variance $D^{2}(X)=\sigma^{2}$, standard deviation $D(X)=\sigma$

non-centered moments $M_{k}(X)$, centered moments $m_{k}(X)$, generating function $g(t)$

characteristic function $c(t)$ etc.

Here we mention only the formulas for mean value, variance and standard deviation: $M(X)=x_{1} p_{1}+x_{2} p_{2}+\cdots x_{n} p_{n}$

$$
D^{2}(X)=M\left(X^{2}\right)-[M(X)]^{2}, D(X)=\sqrt{D^{2}(X)} .
$$

\section{Centralization Table for $n=1,2,3,4,5$}

Application 9. We put toghether all numerical results in a centralization table

Centralization table for $n=1,2,3,4,5$ 


\begin{tabular}{|c|c|c|c|c|c|c|c|c|c|c|c|c|c|c|c|c|}
\hline & $n=1$ & $n=2$ & & $n=3$ & & & $n=4$ & & & & $n=5$ & & & & & \\
\hline & PF & PF & & PF & & & PF & & & & PF & & & & & \\
\hline & I & I & II & I & II & III & I & II & III & IV & I & II & III & IV & $\mathbf{V}$ & \\
\hline $\mathrm{s}=1$ & 1 & 1 & 0 & 1 & 0 & 0 & 1 & 0 & 0 & 0 & 1 & 0 & 0 & 0 & 0 & 1 \\
\hline $\mathrm{s}=2$ & & 1 & 1 & 2 & 1 & 0 & 3 & 1 & 0 & 0 & 4 & 1 & 0 & 0 & 0 & 2 \\
\hline$s=3$ & & & & 1 & 2 & 0 & 3 & 3 & 0 & 0 & 6 & 4 & 0 & 0 & 0 & 3 \\
\hline$s=4$ & & & & 2 & 2 & 2 & 6 & 3 & 3 & 0 & 12 & 4 & 4 & 0 & 0 & 4 \\
\hline $\mathrm{s}=5$ & & & & & & & 1 & 3 & 0 & 0 & 4 & 6 & 0 & 0 & 0 & 5 \\
\hline$s=6$ & & & & & & & 3 & 6 & 3 & 0 & 12 & 12 & 6 & 0 & 0 & 6 \\
\hline$s=7$ & & & & & & & 3 & 3 & 6 & 0 & 12 & 6 & 12 & 0 & 0 & 7 \\
\hline$s=8$ & & & & & & & 6 & 6 & 6 & 6 & 24 & 12 & 12 & 12 & 0 & 8 \\
\hline$s=9$ & & & & & & & & & & & 1 & 4 & 0 & 0 & 0 & 9 \\
\hline $\mathrm{s}=10$ & & & & & & & & & & & 4 & 12 & 4 & 0 & 0 & 10 \\
\hline $\mathrm{s}=11$ & & & & & & & & & & & 6 & 12 & 12 & 0 & 0 & 11 \\
\hline $\mathrm{s}=12$ & & & & & & & & & & & 12 & 24 & 12 & 12 & 0 & 12 \\
\hline$s=13$ & & & & & & & & & & & 4 & 4 & 12 & 0 & 0 & 13 \\
\hline$s=14$ & & & & & & & & & & & 12 & 12 & 24 & 12 & 0 & 14 \\
\hline$s=15$ & & & & & & & & & & & 12 & 12 & 12 & 24 & 0 & 15 \\
\hline$s=16$ & & & & & & & & & & & 24 & 24 & 24 & 24 & 24 & 16 \\
\hline $\mathrm{FF}$ & 1 & 2 & 1 & 6 & 5 & 2 & 26 & 25 & 18 & 6 & 150 & 149 & 134 & 84 & 24 & \\
\hline $\mathrm{S}(\mathrm{n})$ & 1 & - & 3 & - & - & 13 & - & - & & 75 & & & & & 541 & \\
\hline
\end{tabular}

\section{Numerical Examples with Statistical Computations}

Application 10. Let $n=5$ be the number of runners $A, B, C, D, E$.

a) Attach the random variable $X(5), \alpha=100$.

b) Compute the mean value, variance and standard deviation..

Solution. a) We use the values from centralization table

$$
\begin{aligned}
& N(5)=16, S(n)=S(5)=541 \\
& F F 5 I=150, F F 5 I I=149, F F 5 I I I=134, F F 5 I V=84, F F 5 V=24 \\
& X(5)=\left(\begin{array}{ccccc}
100 & 50 & 33.33 & 25 & 20 \\
\frac{150}{541} & =0.277 \frac{141}{541}=0.275 \frac{134}{541}=0.247 \frac{84}{541}=0.155 \frac{24}{541}=0.044
\end{array}\right)
\end{aligned}
$$

b) Compute the mean value, variance and standard deviation.

$M[X(5)]=54.525$ points

$$
X(5)^{2}=\left(\begin{array}{ccccc}
10000 & 2500 & 1111.115 & 625 & 400 \\
0.277 & 0.275 & 0.247 & 0.155 & 0.044
\end{array}\right)
$$

$M\left[X(5)^{2}\right]=3851.5058$ points

$$
D^{2}[X(5)]=3851.5058-2972.9756=878.5302
$$

$D[X(5)]=29.64$ points.

Observation 5. For $n=3,4,5,6$ we have the following statistical results.

$$
M[X(3)]=70.5067 ; M[X(4)]=61.67 ; M[X(6)]=49.2354 .
$$

If the number $n$ is increasing, then the mean value is a decreasing function.

$$
D[X(3)]=27.8604 ; D[X(4)]=28.5303 ; D[X(6)]=30.601 \text {. }
$$

If the number $n$ is increasing, then the standard deviation of score is an increasing function,

\section{Work Hypothesis 2 and Its Implications}

Work hypothesis 2 . Again, a number of $n$ competitors run in order to reach on the arrival line $L[2,10]$. We suppose that 
only one runner could ocuppy each place.

Hence, there is only one non-nominal state $(1,1,1, \cdots, 1)$ and there is $L(n)$ nominal states. We have $L(n)=n !$.

Application 11.

\begin{tabular}{|c|c|c|c|c|}
\hline$n=1$ & $n=2$ & $n=3$ & $n=4$ & $n=5$ \\
\hline I & I II & I II III & I II III IV & I II III IV V \\
\hline \multirow[t]{11}{*}{ (A) } & $(\mathrm{A}, \mathrm{B})$ & $(\mathrm{A}, \mathrm{B}, \mathrm{C})$ & $(\mathrm{A}, \mathrm{B}, \mathrm{C}, \mathrm{D})$ & $(\mathrm{A}, \mathrm{B}, \mathrm{C}, \mathrm{D}, \mathrm{E})$ \\
\hline & $(\mathrm{B}, \mathrm{A})$ & $(\mathrm{A}, \mathrm{C}, \mathrm{B})$ & $(\mathrm{A}, \mathrm{B}, \mathrm{D}, \mathrm{C})$ & $(\mathrm{A}, \mathrm{B}, \mathrm{C}, \mathrm{E}, \mathrm{D})$ \\
\hline & $L(2)$ & $(\mathrm{B}, \mathrm{A}, \mathrm{C})$ & $(\mathrm{A}, \mathrm{C}, \mathrm{B}, \mathrm{D})$ & $(\mathrm{A}, \mathrm{B}, \mathrm{D}, \mathrm{C}, \mathrm{E})$ \\
\hline & & $(\mathrm{B}, \mathrm{C}, \mathrm{A})$ & $(\mathrm{A}, \mathrm{C}, \mathrm{D}, \mathrm{B})$ & $(\mathrm{A}, \mathrm{B}, \mathrm{D}, \mathrm{E}, \mathrm{C})$ \\
\hline & & $(\mathrm{C}, \mathrm{A}, \mathrm{B})$ & $(\mathrm{A}, \mathrm{D}, \mathrm{B}, \mathrm{C})$ & $(\mathrm{A}, \mathrm{B}, \mathrm{E}, \mathrm{C}, \mathrm{D})$ \\
\hline & & $(\mathrm{C}, \mathrm{B}, \mathrm{A})$ & $(\mathrm{A}, \mathrm{D}, \mathrm{C}, \mathrm{B})$ & $(\mathrm{A}, \mathrm{B}, \mathrm{E}, \mathrm{D}, \mathrm{C})$ \\
\hline & & $L(3)=6$ & $(\mathrm{~B}, \mathrm{C}, \mathrm{D}, \mathrm{A})$ & $(\mathrm{A}, \mathrm{C}, \mathrm{D}, \mathrm{E}, \mathrm{B})$ \\
\hline & & & $(B, C, A, D)$ & $(\mathrm{A}, \mathrm{C}, \mathrm{D}, \mathrm{B}, \mathrm{E})$ \\
\hline & & & $L(4)=24$ & $(\mathrm{~A}, \mathrm{C}, \mathrm{E}, \mathrm{D}, \mathrm{B})$ \\
\hline & & & & etc. \\
\hline & & & & $L(5)=120$ \\
\hline
\end{tabular}

Application 12. We define the following random variables, with $\alpha=100$ points.

$Y(3), Y(4), Y(5), Y(6)$ and calculate the main numerical characteristics.

$$
\begin{aligned}
& Y(3)=\left(\begin{array}{ccc}
I & I I & I I I \\
\frac{F F 3 I}{L(3)} & \frac{F F 3 I I}{L(3)} & \frac{F F 3 I I I}{L(3)}
\end{array}\right)=\left(\begin{array}{ccc}
100 & 50 & 33.33 \\
\frac{2}{6} & \frac{2}{6} & \frac{2}{6}
\end{array}\right) \\
& Y(4)=\left(\begin{array}{cccc}
I & I I & I I I & I V \\
\frac{F F 4 I}{L(4)} & \frac{F F 4 I I}{L(4)} & \frac{F F 4 I I I}{L(4)} & \frac{F F 4 I V}{L(4)}
\end{array}\right)=\left(\begin{array}{cccc}
100 & 50 & 33.33 & 25 \\
\frac{6}{24} & \frac{6}{24} & \frac{6}{24} & \frac{6}{24}
\end{array}\right) \\
& Y(5)=\left(\begin{array}{ccccc}
I & I I & I I I & I V & V \\
\frac{F F 5 I}{L(5)} & \frac{F F 5 I I}{L(5)} & \frac{F F 5 I I I}{L(5)} & \frac{F F 5 I V}{L(5)} & \frac{F F 5 V}{L(5)}
\end{array}\right)=\left(\begin{array}{ccccc}
100 & 50 & 33.33 & 25 & 20 \\
\frac{24}{120} & \frac{24}{120} & \frac{24}{120} & \frac{24}{120} & \frac{24}{120}
\end{array}\right) \\
& Y(6)=\left(\begin{array}{cccccc}
I & I I & I I I & I V & V & V I \\
\frac{F F 6 I}{L(6)} & \frac{F F 6 I I}{L(6)} & \frac{F F 6 I I I}{L(6)} & \frac{F F 6 I V}{L(6)} & \frac{F F 6 V}{L(6)} & \frac{F F 6 V I}{L(6)}
\end{array}\right)=\left(\begin{array}{cccccc}
100 & 50 & 33.33 & 25 & 20 & 16.66 \\
\frac{120}{720} & \frac{120}{720} & \frac{120}{720} & \frac{120}{720} & \frac{120}{720} & \frac{120}{720}
\end{array}\right) .
\end{aligned}
$$

Mean value of $Y$.

$$
\begin{gathered}
M[Y(3)]=61.11, M[Y(4)]=52.1, M[Y(5)]=45.7, M[Y(6)]=40.8 \text { points. } \\
\text { Mean value of } Y^{2} . M\left[Y(3)^{2}\right]=4537 \\
M\left[Y(4)^{2}\right]=3559, M\left[Y(5)^{2}\right]=2927.2, M\left[Y(6)^{2}\right]=2486
\end{gathered}
$$

Variance.

$$
D^{2} X(3)=802.6, D^{2} X(4)=844.6, D^{2} X(5)=838.5, D^{2} X(6)=821
$$

Standard deviation

$$
D X(3)=28.33, D X(4)=29.1, D X(5)=28.96, D X(6)=28.65 \text { points }
$$

\section{Conclusions}

This work ilustrates all elements of a study subject: formulates the problem and solve it. Any reader can use it immediately. The work could have a practical application in sport competitions.

Then, combinatorial problem and the statistical problem 
generates an informatics model and a codification program.

In our days there exists very powerful devices to count the exact time for any runner on arrival line. That is why the hypothesis 1 remains as a nice mathematical case.

\section{References}

[1] Popoviciu Nicolae, (2017). A Combinatorial Problem for Possible States on the Arrival Line for n Competitor Runners; Proceedings of ENEC Confference, Editor in Chief Ion Spanulescu; May 2017, Hyperion University, Bucharest, Romania.

[2] Popoviciu Nicolae, (2014). Capitole fundamentale de probabilităţi și statistică matematică; (Special Chapters of Probability and Statistics). Editura Victor, Universitatea Hyperion din București, ISBN 978-973-1815-98-5.

[3] Bona, Miklos; (2005). A Walk Through Combinatorics: An
Itroduction to Enumeration and Graph Theory; World Scientific Publishing; ISBN 981-02-49000-4.

[4] Nathanson M, B; (2000). Elementary Methods in Number Theory; 195; Springer-Verlag; ISBN 0-387-98912-9.

[5] Riordan, J.; (1958), An Introduction to Combinatorial Analysis, Wiley.

[6] Hall, M., (1967), Combinatorial Theory, Blaisdell.

[7] Beckenbach, E. F.(ed); (1964), Applied Combinatorial Mathematics, Wiley.

[8] Erdos, P.; (1974), Probabilistic Methods in Combinatorics, Acad. Press.

[9] Sachov, V. N.; (1977), Combinatorial Methods in Discrete Mathematics, Moscow (in Russian).

[10] Sachov, V. N.; (1978), Probabilistic Methods in Combinatorial Analysiss, Moscow (in Russian). N Popoviciu Revised 24 May 2021. 